\title{
MODELAGEM MATEMÁTICA E TECNOLOGIAS DIGITAIS EDUCACIONAIS: POSSIBILIDADES E APROXIMAÇÕES POR MEIO DE UMA REVISÃO SISTEMÁTICA DE LITERATURA
}

\section{MATHEMATICAL MODELLING AND EDUCATIONAL DIGITAL TECHNOLOGIES: POSSIBILITIES AND APPROACHES IN A SYSTEMATIC LITERATURE REVIEW}

\author{
Rudolph dos Santos Gomes Pereira \\ Universidade Estadual do Norte do Paraná/Departamento de Matemática/ \\ rudolphsantos@uenp.edu.br \\ Jeferson Takeo Padoan Seki \\ Universidade Estadual do Norte do Paraná/Departamento de Matemática/ \\ jefersontakeopadoanseki@hotmail.com \\ Bárbara Nivalda Palharini \\ Universidade Estadual do Norte do Paraná/Departamento de Matemática/ \\ barbara.palharini@uenp.edu.br \\ João Coelho Neto \\ Universidade Estadual do Norte do Paraná/Departamento de Matemática/ \\ joaocoelho@uenp.edu.br
}

\section{Ariel Cardoso da Silva}

Universidade Estadual do Norte do Paranál ariel.c.silva@live.com

\section{Willian Damin}

Universidade Estadual do Norte do Paraná/Departamento de Matemática/ wdamin@uenp.edu.br

\section{Bianca de Oliveira Martins}

Universidade Estadual de Londrina/ bianca o.martins@hotmail.com

\section{Resumo}

Este artigo apresenta um mapeamento sobre a Modelagem Matemática e Tecnologias Digitais em Teses e Dissertações resultantes de programas de Pós-Graduação, com notas 5, 6 e 7, conceituados pela Coordenação de Aperfeiçoamento Pessoal de Nível Superior, de janeiro de 2010 a maio de 2016, em consultas aos sites dos programas. A fim de responder a questão de pesquisa: Quais são as Tecnologias Digitais Educacionais utilizadas no desenvolvimento de atividades de Modelagem Matemática nos processos de ensino e de aprendizagem de Matemática? os encaminhamentos metodológicos seguiram os pressupostos de uma Revisão Sistemática de Literatura por meio de dois momentos. Inicialmente foram selecionadas as produções referentes Modelagem Matemática na 
Educação Matemática e posteriormente uma síntese foi elaborada em relação ao uso de tecnologias digitais educacionais nas produções pesquisadas. Os resultados apontam para diferentes usos da Internet e de softwares educacionais em atividades de Modelagem Matemática.

Palavras-chave: Educação Matemática; Modelagem Matemática; Tecnologias Digitais Educacionais; Revisão Sistemática de Literatura; Teses e Dissertações.

\section{Abstract}

This paper presents a mapping of the Mathematical Modelling and Digital Technologies regarding Theses and Dissertations resulting from Graduate programs with concepts 5, 6 and 7 according Coordination Higher Education Personnel, the search was made from January 2010 to May 2016 in the graduate program websites. To answer the research question: what are the Educational Digital Technologies used to develop mathematical modelling activities in the mathematics' teaching and learning? methodological approach followed the assumptions of a Systematic Review of Literature in two moments. Initially productions were selected regarding Mathematical Modelling in Mathematics Education, and later a synthesis was drawn up in relation to the use of digital technologies in education researched productions. The results point to different uses of the Internet and educational software during mathematical modelling activities.

Keywords: Mathematics Education; Mathematical Modelling; Educational Digital Technologies; Systematic Literature Review; Theses and Dissertations.

\section{Introdução}

A presença das Tecnologias Digitais de Informação e Comunicação (TDIC) na sociedade contemporânea, com atuações em diversas áreas, vêm provocando mudanças no mercado de trabalho, na pesquisa científica e consequentemente no cotidiano das pessoas. Nesse sentido, não se pode ignorar as potencialidades do uso das tecnologias nos processos de ensino e de aprendizagem, como expõe as Orientações Curriculares para o Ensino Médio (BRASIL, 2006, p. 87).

Não se pode negar o impacto provocado pela tecnologia de informação e comunicação na configuração da sociedade atual. Por um lado, tem-se a inserção dessa tecnologia no dia-a-dia da sociedade, a exigir indivíduos com capacitação para bem usá-la; por outro lado, tem-se nessa mesma tecnologia um recurso que pode subsidiar o processo de aprendizagem da Matemática. É importante contemplar uma formação escolar nesses dois sentidos, ou seja, a Matemática como ferramenta para entender a tecnologia, e a tecnologia como ferramenta para entender a Matemática.

O mesmo documento sinaliza o trabalho com a Modelagem Matemática, numa perspectiva voltada para a Educação Matemática, como meio para desenvolver competências e habilidades inerentes a formação do estudante. A cidadania ambiental 
deve ser discutida nas abordagens atuais do ensino fundamental e médio para que os estudantes possam desenvolver sua capacidade de análise crítica (SANTOS, 2001).

Ante uma situação-problema ligada ao "mundo real", com sua inerente complexidade, 0 aluno precisa mobilizar um leque variado de competências: selecionar variáveis que serão relevantes para o modelo a construir; problematizar, ou seja, formular o problema teórico na linguagem do campo matemático envolvido; formular hipóteses explicativas do fenômeno em causa; recorrer ao conhecimento matemático acumulado para a resolução do problema formulado [...]; validar, isto é, confrontar as conclusões teóricas com os dados empíricos existentes; e eventualmente ainda, quando surge a necessidade, modificar o modelo para que esse melhor corresponda à situação real, aqui se revelando o aspecto dinâmico da construção do conhecimento (BRASIL, 2006, p. 85).

Neste artigo, constitui-se como tema a Modelagem Matemática e as Tecnologias Digitais Educacionais, assim, buscou-se na literatura possíveis relações entre estas duas áreas, a fim de subsidiar o desenvolvimento da pesquisa aqui relatada. No âmbito da Educação Matemática a Modelagem Matemática, enquanto linha de pesquisa, tem se apresentado com área de interesse de pesquisadores e educadores. De fato, são diversas as publicações em eventos (BEAN, 2015, BONOTTO; SCHELLER; BIEMBENGUT, 2015, SEKI; SILVA; PEREIRA, 2016, entre outros), periódicos (ALMEIDA; PALHARINI, 2012, BARBOSA, 2004, BISOGNIN; BISOGNIN, 2012, PEREIRA; SANTOS JÚNIOR, 2013, entre outros) e livros (ALMEIDA; SILVA; VERTUAN, 2012, BASSANEZI, 2002, BRANDT; BURAK, KLÜBER, 2010, entre outros). Em meio a este volume de pesquisas, algumas têm se voltado para as Tecnologias Digitais Educacionais associadas a Modelagem Matemática tendo como exemplo os trabalhos de Araújo (2002), Borba e Chiari (2014), Malheiros (2004), entre outros.

Dado o corpo continuamente crescente de estudos nas temáticas, esta pesquisa visa investigar nos programas stricto-sensu trabalhos que abordam Modelagem Matemática na Educação Matemática e, nestes, as Tecnologias Digitais Educacionais. Essa interlocução tem como finalidade vislumbrar quais são as tecnologias educacionais utilizados em conjunto com a Modelagem Matemática, em particular, a fim de contribuir com os processos de ensino e de aprendizagem de Matemática. A pesquisa consistiu de uma revisão sistemática de literatura que considerou teses de doutorado e dissertações de mestrado publicadas no período de janeiro de 2010 a maio de 2016.

O levantamento foi realizado com base nas teses e dissertações defendidas e disponibilizadas nas páginas dos programas de pós-graduação stricto-sensu da área de Ensino que obtiveram conceitos 5, 6 e 7, de acordo com avaliação da Coordenação de Aperfeiçoamento de Pessoal de Nível Superior (CAPES), no período supracitado.

Dessa forma, visando analisar as características acima descritas, este artigo tem como objetivo mapear quais são as Tecnologias Digitais Educacionais utilizadas nas teses e dissertações que tratam da Modelagem Matemática na Educação Matemática e analisar quais dessas tecnologias são utilizadas nos processos de ensino e aprendizagem de Matemática. Este artigo compreende cinco seções: a primeira seção, contextualiza a temática; na segunda, o aporte teórico; na terceira, os encaminhamentos metodológicos 
utilizados; na quarta, a análise empreendida e os resultados obtidos; na quinta, as considerações finais.

\section{Modelagem Matemática e Tecnologias Digitais Educacionais}

A parceria entre Modelagem Matemática e as Tecnologias Digitais Educacionais pode se dar de forma harmônica, pois, de acordo com Araújo (2002), parece existir um convite natural pelo uso das tecnologias ao se desenvolver atividades de modelagem matemática.

Dada a possibilidade de relacionar estas duas áreas, busca-se nesta seção contextualizar o leitor sobre a Modelagem Matemática, bem como seus entendimentos, fases e contribuições para os processos de ensino e de aprendizagem de Matemática. Em seguida, apresentam-se alguns aspectos da relação entre Modelagem Matemática e as Tecnologias Digitais Educacionais.

De modo geral, as raízes da Modelagem Matemática estão ancoradas na Matemática Aplicada, em que os "modeladores" a utilizam em processos de previsão, otimização e representação de fenômenos naturais, físicos, químicos, entre outros, por meio de modelos matemáticos (BASSANEZI, 2002). No âmbito da Educação Matemática, a Modelagem Matemática pode ser entendida como "uma alternativa pedagógica na qual fazemos uma abordagem, por meio da Matemática, de uma situação-problema não essencialmente Matemática" (ALMEIDA; SILVA; VERTUAN, 2012, p. 17).

De acordo com Almeida, Silva e Vertuan (2012) uma atividade de modelagem matemática envolve uma situação inicial (problemática) e uma situação final (resposta para a problemática) e um conjunto de procedimentos, tais como a escolha do tema, coleta de dados, simplificação, formulação do problema, seleção de variáveis, obtenção de um modelo matemático, validação e interpretação dos resultados, conforme a Figura 1.

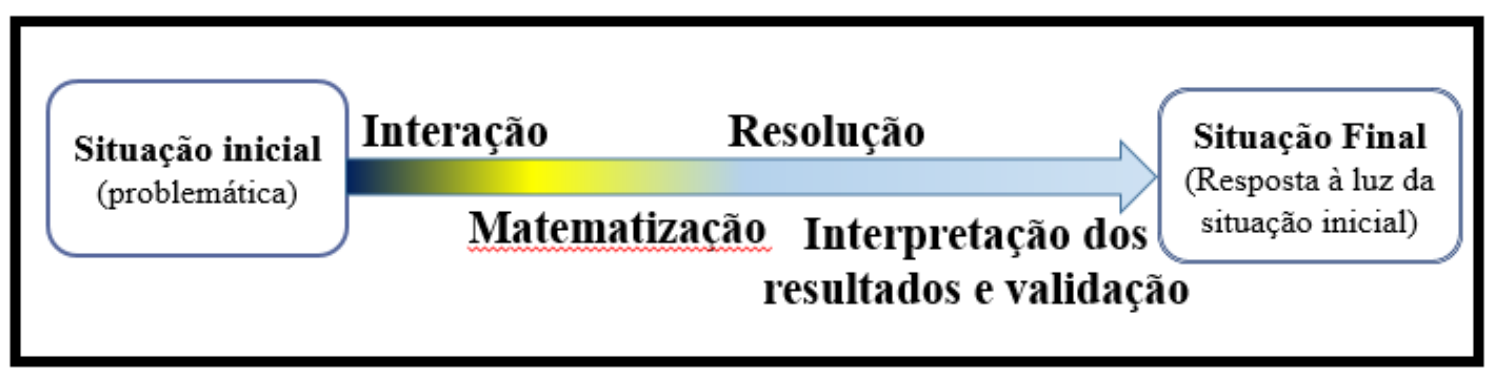

Figura 1 - Fases da Modelagem Matemática

Fonte - Adaptado de Almeida, Silva e Vertuan (2012, p. 15)

$\mathrm{Na}$ literatura, diversas são as potencialidades da Modelagem Matemática, ressaltadas por pesquisadores, para os processos de ensino e de aprendizagem. Bassanezi (2002) apresenta seis argumentos para sua inserção no ensino de Matemática: formativo, de competência crítica, de utilidade, intrínseco, de aprendizagem, de alternativa epistemológica. Tais argumentos, estão relacionados ao desenvolvimento de competências, habilidades e da criatividade, ao aprimoramento da compreensão de argumentos matemáticos e conceitos matemáticos e valorização da própria matemática.

Barbosa (2004, p. 2) argumenta que a Modelagem Matemática pode promover "a intervenção das pessoas nos debates sociais que envolvem aplicações da matemática, o 
que me parece ser uma contribuição para alargar as possibilidades de construção e consolidação de sociedades democráticas".

Segundo Vertuan (2007, p. 131) o desenvolvimento de atividades de modelagem matemática coloca os alunos "em um contexto de aprendizagem em que a discussão de situações-problema, a participação ativa e o uso de diferentes registros se fazem essenciais".

No âmbito das salas de aula, as atividades de modelagem matemática podem ser trabalhadas com o auxílio de outras tendências metodológicas (PARANÁ, 2008), como, por exemplo, as Tecnologias Digitais Educacionais que vem com o "intuito de tentar resolver problemas que envolvem a investigação e a experimentação-com-tecnologias, que são muito valorizados e discutidos na Educação Matemática" (MALHEIROS, 2004, p. 53).

Almeida, Silva e Vertuan (2012) destacam diversas razões para o uso das Tecnologias Digitais Educacionais, principalmente as informáticas, em consonância com a Modelagem Matemática.

A dinamicidade de inúmeros softwares livres, hoje disponíveis no mercado, pode auxiliar alunos e professor na construção de gráficos e na observação da influência dos parâmetros bem como na realização de cálculos. Nesse sentido, a possibilidade de experimentar, de visualizar e de coordenar de forma dinâmica as representações algébricas, gráficas e tabulares, são vantagens da interação de atividades de modelagem com as mídias informáticas (ALMEIDA; SILVA; VERTUAN, 2012, p. 31).

Com base no que foi exposto, pode-se notar que quando a Modelagem Matemática é trabalhada de forma articulada com as TDIC diferentes contribuições são possíveis no que se refere ao ensino e à aprendizagem de Matemática. Diante disso, neste trabalho buscou-se identificar quais tipos de Tecnologias Digitais Educacionais têm sido utilizadas em atividades de modelagem matemática em Teses e Dissertações produzidas nos últimos seis anos e veiculadas pela CAPES e quais são utilizadas nos processos de ensino e de aprendizagem de Matemática.

\section{Encaminhamentos Metodológicos}

Nesta pesquisa utilizou-se de uma adaptação da Revisão Sistemática de Literatura de Kitchenham (2004) como um meio de averiguar, avaliar e interpretar as pesquisas disponíveis relevantes para uma determinada pergunta de pesquisa, área de um tópico, ou fenômeno de interesse, produzidas em determinado período.

A condução da revisão foi embasada nas fases determinadas pela autora: 1 . Identificação de pesquisa; 2. Seleção de estudos primários; 3. Estudo da avaliação de qualidade; 4. Extração e monitorização de dados 5. Síntese de dados.

$\mathrm{Na}$ identificação de pesquisa, gerou-se a seguinte pergunta norteadora: quais são as Tecnologias Digitais Educacionais utilizadas no desenvolvimento de atividades de modelagem matemática no processo de ensino e de aprendizagem de Matemática? 
Para a seleção dos estudos primários, utilizou-se o site da CAPES para determinação dos programas stricto-sensu na área de Ensino que obtiveram notas 5, 6, e 7, considerados estes como excelência. Uma vez selecionado os programas, mapeou-se as dissertações e teses disponíveis na página de cada curso, com base em dois momentos:

Primeiro momento: Consistiu da seleção de produções científicas que possuíam no título, resumo e/ou palavras-chave os termos, "Modelagem"; "Modelagem Matemática"; "Modelo"; "Modelo Matemático"; "Modelização"; "Modelação"; "Matematização". As escolhas dos termos em relação a Modelagem Matemática se deram devido as diversas definições presentes na literatura como as de Almeida, Silva e Vertuan (2012), Barbosa (2004), Biembengut (2016), Burak (1992, 2004), entre outras.

- Segundo momento: a partir da leitura de cada resumo das produções pesquisadas, foram selecionados os trabalhos que possuíam como enfoque temático a Tecnologia e a Modelagem Matemática no âmbito da Educação Matemática a partir dos termos de busca, "Tecnologia", "Tecnologias Digitais", "ambiente computacional", "softwares", nos títulos, resumos e/ou palavras-chave dos dados.

$\mathrm{Na}$ síntese dos resultados, foi utilizado como critério de inclusão, os trabalhos que abordavam o uso das TDIC no desenvolvimento de atividades de modelagem matemática numa perspectiva voltada para os processos de ensino e de aprendizagem de Matemática e áreas afins.

O Estudo e Avaliação e a Extração e monitoração dos dados, bem como a validação dos resultados foram feitos pelos participantes do grupo de pesquisa Grupo de Pesquisa em Ensino de Matemática (GEPIEEM) na tentativa de explorar os resultados e revisar os protocolos.

A Síntese dos dados foi composta pela articulação de quais são os instrumentos digitais educacionais utilizados com a Modelagem Matemática, a fim de auxiliar no processo de ensino e aprendizagem da Matemática.

\section{Discussão dos Resultados}

No intuito de atender o objetivo deste trabalho, identificou-se os programas de pósgraduação stricto-sensu consultados e suas respectivas regiões - Tabela 1. A partir do mapeamento dos programas de pós-graduação consultados, codificações foram estruturadas para facilitar o processo de análise dos dados: M: Mestrado Acadêmico; MP: Mestrado Profissional; D: Doutorado. 
Tabela 1 - Mapeamento dos Programas de Pós-Graduação

\begin{tabular}{|c|c|c|c|c|c|c|}
\hline \multicolumn{7}{|c|}{ Área de consulta: Ensino } \\
\hline Número & IES/campus & $\begin{array}{l}\text { Programa de pós- } \\
\text { graduação }\end{array}$ & Curso & Nota & UF & Região \\
\hline 1 & UNESP/BAU & $\begin{array}{l}\text { Educação para a } \\
\text { ciência }\end{array}$ & M; D & 6 & SP & Sudeste \\
\hline 2 & UNESP/RC & $\begin{array}{l}\text { Educação } \\
\text { Matemática }\end{array}$ & M; D & 6 & SP & Sudeste \\
\hline 3 & UEL & $\begin{array}{c}\text { Ensino de Ciências } \\
\text { e Educação } \\
\text { Matemática }\end{array}$ & M; D & 6 & PR & Sul \\
\hline 4 & UFSC & $\begin{array}{c}\text { Educação Científica } \\
\text { e Tecnológica }\end{array}$ & M; D & 6 & SC & Sul \\
\hline 5 & FIOCRUZ & $\begin{array}{c}\text { Ensino em } \\
\text { Biociências e Saúde }\end{array}$ & M; D & 5 & RJ & Sudeste \\
\hline 6 & PUC/SP & $\begin{array}{l}\text { Educação } \\
\text { Matemática }\end{array}$ & $\begin{array}{c}\mathrm{M} ; \\
\mathrm{MP} ; \mathrm{D}\end{array}$ & 5 & SP & Sudeste \\
\hline 7 & UNIBAN & $\begin{array}{l}\text { Educação } \\
\text { Matemática }\end{array}$ & M; D & 5 & SP & Sudeste \\
\hline \multirow[b]{2}{*}{8} & \multirow[b]{2}{*}{ UNICSUL } & Ensino de Ciências & $\mathrm{M} ; \mathrm{D}$ & \multirow[b]{2}{*}{5} & \multirow[b]{2}{*}{ SP } & \multirow[b]{2}{*}{ Sudeste } \\
\hline & & $\begin{array}{c}\text { Ensino de Ciências } \\
\text { e Matemática }\end{array}$ & $\mathrm{MP} ; \mathrm{D}$ & & & \\
\hline 9 & USP & $\begin{array}{l}\text { Ensino de Ciências } \\
\text { (Modalidades } \\
\text { Físicas, Químicas e } \\
\text { Biológicas) }\end{array}$ & M; D & 5 & SP & Sudeste \\
\hline 10 & UNICAMP & $\begin{array}{c}\text { Ensino e História de } \\
\text { Ciências da Terra }\end{array}$ & M; D & 5 & SP & Sudeste \\
\hline 11 & UFBA & $\begin{array}{c}\text { Ensino, Filosofia e } \\
\text { História das } \\
\text { Ciências }\end{array}$ & M; D & 5 & $\mathrm{BA}$ & Nordeste \\
\hline 12 & UFRJ & $\begin{array}{c}\text { Educação em } \\
\text { Ciências e Saúde }\end{array}$ & M; D & 5 & RJ & Sudeste \\
\hline \multirow[t]{3}{*}{13} & \multirow[t]{3}{*}{ UFRGS } & $\begin{array}{l}\text { Educação em } \\
\text { Ciências Química } \\
\text { da vida e saúde } \\
\text { (UFSM - FURG) }\end{array}$ & M; D & \multirow[t]{3}{*}{5} & \multirow[t]{3}{*}{ RS } & \multirow[t]{3}{*}{ Sul } \\
\hline & & Ensino de Física & $\mathrm{M} ; \mathrm{D}$ & & & \\
\hline & & Ensino de Física & MP & & & \\
\hline
\end{tabular}

Fonte: CAPES. Acesso em: 11 ago. 2016.

A partir da identificação das instituições, dados foram coletados no que tange a Modelagem Matemática na Educação Matemática e posteriormente referente as Tecnologias Digitais Educacionais nas produções sobre Modelagem Matemática, por 
meio da leitura do título, resumo e palavras-chave de cada produção. Obteve-se o total de quarenta e nove trabalhos no primeiro momento e oito no segundo momento. Entretanto, após realizar a síntese dos dados, notou-se que apenas cinco dessas oito teses e dissertações apresentavam o uso das Tecnologias Digitais Educacionais (TDE) como elemento importante para o desenvolvimento das atividades de modelagem matemática Tabela 2.

Tabela 2 - Trabalhos Mapeados

\begin{tabular}{|c|c|}
\hline CÓDIGO1 & TÍTULO \\
\hline 2_D_12_1 & $\begin{array}{c}\text { A MODELAGEM MATEMÁTICA E A REALIDADE DO MUNDO } \\
\text { CIBERNÉTICO }\end{array}$ \\
\hline 2_D_12_2 & $\begin{array}{c}\text { UMA ABORDAGEM PEDAGÓGICA BASEADA NA ANÁLISE DE } \\
\text { MODELOS PARA ALUNOS DE BIOLOGIA: QUAL O PAPEL DO } \\
\text { SOFTWARE? }\end{array}$ \\
\hline 3_D_13_3 & $\begin{array}{r}\text { MODELAGEM MATEMÁTICA, A APRENDIZAGEM } \\
\text { DIFERENTES CONTEXTOS EDUCACIONAIS }\end{array}$ \\
\hline 6_MP_11_5 & $\begin{array}{r}\text { A MODELAGEM MATEMÁTICA NA INTRODUÇÃO DO } \\
\text { CONCEITO DE EQUAÇO PARA ALUNOS DE SÉTIMO ANO DO } \\
\text { ENSINO FUNDAMENTAL }\end{array}$ \\
\hline 6_D_15_6 & $\begin{array}{r}\text { PARAMETRIZAÇÃO E MOVIMENTAÇÃO DE CURVAS E } \\
\text { SUPERFÍCIES PARA USO EM MODELAÇÃO MATEMÁTICA }\end{array}$ \\
\hline
\end{tabular}

Fonte: Os autores.

A tese 2_D_12_1 apresenta o uso das TDE não somente como mediadora no trabalho com Modelagem Matemática, mas também considera que o uso das TDE pode ser feito na finalidade de atualizarem a construção dos modelos no mundo cibernético, isto é, um mundo intermediado pela construção de jogos eletrônicos. Nesta tese, o autor apresenta dois softwares utilizados pelos alunos o Scratch2 e o Autodesk 3Ds3, que permitem a construção de jogos eletrônicos e a criação de animações.

As análises foram realizadas em torno de quatro categorias, a saber, objetivo pedagógico, modelo, problema e referência a realidade. No que diz respeito ao objetivo pedagógico, o autor aponta: a) a possiblidade de abranger aspectos relacionados a linguagem natural verbalizada e características peculiares a linguagem utilizada na programação; b) a possibilidade de ampliar ideias já existentes, envolvendo uma

\footnotetext{
1 O código estabelecido para nomear os trabalhos selecionados segue a sequência: número atribuído ao programa de pós-graduação_modalidade da produção_ano de publicação_número atribuído aos trabalhos em ordem crescente de 1 a 6.

${ }^{2}$ Disponível em: < https://scratch.mit.edu/>.

${ }^{3}$ Disponível em: < http://www.autodesk.com.br/>.
} 
multiplicidade de caminhos que podem ser seguidos durante a atividade; c) Embora a matemática não esteja relacionada de forma direta com os jogos eletrônicos, a reflexão sobre essa construção pode evidenciar aspectos matemáticos. Sinteticamente, as discussões apontaram que o trabalho com Modelagem Matemática entrelaçado ao uso de jogos eletrônicos, considerando o mundo cibernético, ocorre de forma fluida em constante transformação.

Tais resultados vão ao encontro do exposto por Malheiros (2004) que sinaliza o uso de tecnologias digitais para tentar resolver problemas que envolvem a investigação e a experimentação-com-tecnologias, como no caso do uso dos softwares Scratch4 e o Autodesk 3Ds5. Embora as relações com o ensino e a aprendizagem de Matemática não sejam explicitas no que tange o uso da modelagem matemática e a realidade do mundo cibernético, a construção de modelos no mundo cibernético pode se configurar como alternativas para o trabalho com modelos que imitam situações da realidade. $A$ construção destes modelos, por meio do uso destes softwares, pode viabilizar o que Almeida, Silva e Vertuan (2012) denominam como possibilidades de experimentar, de visualizar e de coordenar de forma dinâmica diferentes representações matemáticas.

A tese 2_D_12_2 expõe um estudo realizado com alunos de um curso de ciências biológicas, no qual a pesquisadora propõe a análise de um modelo matemático para um fenômeno biológico por meio do software Modellus6. O problema norteador da pesquisa buscou compreender o papel deste software numa abordagem pedagógica embasada na análise de modelos. Após efetuar as análises, a autora identificou três papéis: proporcionar resultados sobre o fenômeno, colaborar para a compreensão de conceitos matemáticos e intermediar as relações entre as diferentes áreas do conhecimento, neste caso a Matemática e a Biologia.

Em suas considerações finais, a autora destaca a importância de sua tese para a comunidade científica, na medida em que pode colaborar com o debate sobre o papel da tecnologia no processo de produção de conhecimento. Além disso, ressalta as condições ofertadas pela pesquisa para o desenvolvimento do coletivo pensante e para o pensar com a tecnologia.

O foco na análise de modelos pode se configurar, no cenário da Modelagem Matemática na Educação Matemática, como uma perspectiva associada ao uso da modelagem matemática em sala de aula, em particular no que tange ao ensino e a aprendizagem de Matemática. A análise de modelos clássicos, de acordo com Bassanezi (2002), está associada tanto ao conhecimento a respeito do fenômeno quanto com relação ao conhecimento sobre os conceitos e artefatos matemáticos que circundam a elaboração de modelos. Os resultados indicados por 2_D_12_2 corroboram com a literatura da área e enfatizam a importância da análise de modelos para conhecimentos dos fenômenos e de conceitos matemáticos. Pode-se dizer que nesse sentido diversos softwares podem ser apresentados aos alunos como um auxílio na visualização e análise de fenômenos, pois possuem geralmente possuem ferramentas que possibilitam a simulação das situações, permitindo assim, melhor acompanhamento e verificação das

\footnotetext{
${ }^{4}$ Disponível em: < https://scratch.mit.edu/>.

${ }^{5}$ Disponível em: < http://www.autodesk.com.br/>.

${ }^{6}$ Disponível em: < http://modellus.co/index.php?lang=pt >.
} 
possibilidades de associação dos conhecimentos matemáticos as mais diversas situações.

A tese 3_D_13_3 coloca em foco Modelagem Matemática, Aprendizagem Significativa e Tecnologias. A articulação entre estes conceitos é investigada em três contextos educacionais: uma disciplina de Modelagem Matemática na Licenciatura em Matemática, um curso de curta duração e atividades práticas supervisionadas na disciplina de Cálculo Numérico. Dentre os recursos tecnológicos utilizados evidencia-se a Internet, Câmera digital, o uso de softwares como o Maple7, Origin8, Microsoft Excel9, Geogebra10 e o Tracker11. Os usos de tais tecnologias se deram como ferramenta de pesquisa na coleta de dados e na interação dos alunos com o tema, bem como para a produção e análise de dados no decorrer das atividades de modelagem matemática.

Em um tópico intitulado relações entre a tecnologia e seus usos, a autora apresenta algumas contribuições dos recursos tecnológicos para o desenvolvimento das atividades como, por exemplo, a importância do software Tracker, enquanto mediador, para as discussões e definições, colaborando para o pensar juntos com a tecnologia. Outro aspecto evidenciado é o desempenho relevante das Tecnologias Digitais no trabalho com Modelagem Matemática em contextos educacionais, promovendo diferentes representações dos dados e mostrando-se ou não como parceira intelectual, no sentido que pode beneficiar de maneira mais ou menos contundente na viabilização da aprendizagem significativa.

A dissertação 6_MP_11_5 propõe e analisa o desenvolvimento de uma sequência didática para a introdução do conceito de equações no sétimo ano do Ensino Fundamental, lançando mão da Modelagem Matemática. A pesquisa foi desenvolvida em dois ambientes, na sala de aula e no laboratório de informática, o que possibilitou 0 acesso dos alunos ao software Excel, utilizado durante as atividades.

Os resultados da pesquisa sinalizam o aspecto motivacional do laboratório de informática para os alunos nas resoluções das situações-problema propostas, potencializando as discussões sobre as ideias levantadas. O autor, ainda, destaca as TED como um facilitador da aprendizagem em conjunto com a Modelagem Matemática, num contexto educacional.

Embora o software Excel não seja de livre acesso, a pesquisa 6_MP_11_5 aborda o desenvolvimento de uma sequência didática envolvendo modelagem matemática e tecnologias na Educação Básica. Este foco específico a diferencia das demais pesquisas e sinaliza a importância e a necessidade da articulação destas temáticas neste nível de escolaridade. Pois, ao utilizar as tecnologias digitais educacionais no ensino de matemática têm-se mais uma estratégia didática no qual o foco deve ser o conhecimento matemático e não a TED com um fim em si (OLIVEIRA, 2015). Documentos oficiais como Paraná (2008) sinalizam a importância das tecnologias digitais nos processos de ensino e

\footnotetext{
${ }^{7}$ Disponível em: < http://www.maplesoft.com/products/maple/>.

${ }^{8}$ Disponível em: < http://www.originlab.com/Origin>.

${ }^{9}$ Disponível em: < https://products.office.com/pt-br/excel>.

${ }^{10}$ Disponível em: < https://www.geogebra.org/>.

${ }_{11}$ Disponível em: < http://physlets.org/tracker/>.
} 
de aprendizagem, em particular de Matemática, no entanto das teses e dissertações mapeadas apenas essa tem como foco a Educação Básica.

A escola defronta-se com o desafio de se constituir em um tempo e espaço social e, assim, trazer para o seu contexto o imenso oceano de informações que a envolve, no intuito de articular tais informações com os conhecimentos escolares. (...) as tecnologias no espaço escolar precisam ser entendidas em uma perspectiva que extrapola a ideia de ferramentas de auxílio ao ensino, sendo compreendidas e incorporadas pedagogicamente (...) (KENSKI, 2007, p. 46).

Nesse sentido, tem-se como necessária a inserção das TED no ambiente escolar de modo a possibilitar a investigação, a análise e a compreensão de fatos sociais fazendo do ambiente escolar um espaço de articulação entre os conhecimentos a serem ensinados aos alunos e as TED como uma alternativa pedagógica a ser adotada pelos professores.

A tese 6_D_15_6 possui como temática conteúdos matemáticos estudados nas disciplinas de Cálculo Diferencial e Integral, Geometria Analítica e Álgebra Linear, como a parametrização de curvas e superfícies e transformações. O autor propõe diversas atividades numa perspectiva da Modelação Matemática12 em um ambiente computacional para a articulação, sistematização e aplicação dos conteúdos evidenciados. O software apresentado foi o Winplot13, que permite ao aluno realizar representações gráficas bidimensionais e tridimensionais.

O uso do software para a elaboração das atividades, segundo o autor, possibilitou diferentes formas de representar um objeto matemático e apresentou-se com potencial para articular conteúdos matemáticos. Potencialidades estas já identificadas por Vertuan (2007), Malheiros (2004) e Almeida, Silva e Vertuan (2012) em suas pesquisas e que reafirmam a ideia de que o uso das TED deve ser articulado com o conhecimento matemático, permitindo ao aluno fazer simulações e interpretações e sistematizar conceitos a partir das diferentes situações apresentadas em sala de aula.

Para aprimorar o mapeamento e identificação das TDE que foram utilizados com a Modelagem Matemática a fim de auxiliar no contexto educacional foi elaborado o Tabela 3 que visa identificar essas articulações.

Tabela 3 - Mapeamento dos Resultados

\begin{tabular}{|c|l|c|}
\hline CÓDIGO & \multicolumn{1}{|c|}{ AUTOR } & TDE \\
\hline 2_D_12_1 & DELLA VECCHIA (2012) & $\begin{array}{c}\text { Scratch; } \\
\text { Autodesk 3Ds. }\end{array}$ \\
\hline 2_D_12_2 & SOARES (2012) & Modellus. \\
\hline
\end{tabular}

12 O termo Modelação Matemática utilizado pela tese 6_D_15_6 refere-se à perspectiva de Biembengut (2003), na qual segundo o autor da tese "Biembengut faz uma adaptação da Modelagem Matemática para Modelação Matemática, em que o professor pode optar por determinados modelos, fazendo sua recriação em sala de aula, juntamente com os alunos, de acordo com o nível em questão e obedecendo ao currículo inicialmente proposto" (PARANHOS, 2015, p. 10).

${ }^{13}$ Disponível em: < http://math.exeter.edu/rparris/winplot.html>. 


\begin{tabular}{|c|c|c|}
\hline 3_D_13_3 & BORSSOI (2013) & $\begin{array}{c}\text { Internet; } \\
\text { Câmera digital; } \\
\text { Maple; } \\
\text { Origin; } \\
\text { Microsoft Excel; } \\
\text { Geogebra; } \\
\text { Tracker; }\end{array}$ \\
\hline 6_MP_11_5 & SALANDINI (2011) & $\begin{array}{l}\text { Laboratório de } \\
\text { informática; } \\
\text { Microsoft Excel. }\end{array}$ \\
\hline 6_D_15_6 & PARANHOS (2015) & Winplot. \\
\hline
\end{tabular}

Fonte: Os Autores.

Em relação às diferentes Tecnologias Digitais Educacionais abordados pelas teses e dissertações mapeadas, pode-se notar o uso da Internet para fins de pesquisas sobre os temas das atividades, incluindo coleta de dados, e também o uso de softwares, os quais permitiram aos alunos construírem gráficos, tabelas e jogos eletrônicos, realizarem cálculos e discutirem aspectos de conceitos matemáticos de forma dinâmica e crítica.

De modo geral, é possível sinalizar que os softwares são utilizados nos processos de ensino e de aprendizagem de Matemática como suportes para coordenação de diferentes representações dos objetos matemáticos, bem como para construção e análise de modelos matemáticos, gráficos, algébricos ou associados à realidades virtuais que imitam a realidade. $O$ uso de softwares fechados como o Excel poderia ser substituído por softwares livres, para trabalhar com conceitos associados à lógica, à estatística e ao conceito de equações, como evidenciado pela pesquisa mapeada realizada no Ensino Fundamental.

O uso das tecnologias digitais para coleta de informações é algo comum nas pesquisas que abordam a temática, servindo aos alunos para coleta de dados e exploração de temáticas. Quando o foco é o ensino e a aprendizagem de Matemática, é importante atentar-se para o desenvolvimento de atividades de modelagem matemática aliada as tecnologias digitais que extrapolem o uso das mídias tecnológicas apenas para obtenção de informações, visto as potencialidades destes recursos como destacadas por Malheiros (2004), Vertuan (2007), Paraná (2008) e Almeida, Silva e Vertuan (2012).

Nesse sentido, a adoção de TED pode incentivar os alunos a utilizarem-nas na tentativa de analisar e compreender situações e fenômenos presentes no cotidiano como forma de sistematização e aprendizagem de conceitos escolares. Para isso, o professor necessita inserir as TED como um componente pedagógico em sala de aula, independentemente do nível em que atua, percebendo a sua potencialidade no ensino dos conhecimentos disciplinares, neste caso, da Matemática. 


\section{Considerações Finais}

Este artigo visou mapear por meio da revisão sistemática de literatura quais são Tecnologias Digitais Educacionais utilizados junto com a Modelagem Matemática na finalidade de auxiliar o ensino e a aprendizagem de Matemática. Para estruturar esta pesquisa, uma pergunta norteadora foi delineada: quais são as tecnologias digitais educacionais utilizadas para auxiliar a Modelagem Matemática para contextualizar o ensino da Matemática?

Por meio da pesquisa realizada foi possível mapear trabalhos que retratam esta temática com o uso da Internet e de softwares como o Autodesk 3Ds, Geogebra, Microsoft Excel, Modellus, Maple, Origin, Scratch, Tracker, Winplot. Tais softwares são utilizados por alunos e professores em diferentes contextos no que tange ao desenvolvimento de atividades de modelagem matemática.

Convergindo com os estudos de Almeida, Silva e Vertuan (2012), Araújo (2002) e Malheiros (2004) as Tecnologias Digitais Educacionais mencionadas foram utilizadas no intuito de mediar o desenvolvendo de atividades de modelagem matemática, promovendo à construção de jogos eletrônicos, a produção de dados, a interação com elementos das diferentes situações-problema e diversas representações de objetos matemáticos.

Sintetizando as discussões apresentadas, pode-se perceber diversos argumentos favoráveis para o uso das Tecnologias Digitais Educacionais no trabalho com a Modelagem Matemática. Os resultados das pesquisas investigadas apontam elementos como a motivação dos estudantes, potencialização de discussões e para o pensar juntos com a tecnologia, facilitação da aprendizagem, multiplicidades de possibilidades para o envolvimento dos alunos com a resolução das atividades. Tais resultados estão associados ao uso de softwares, em particular, de manipulação de dados algébricos e à visualização de gráficos de funções.

Sinaliza-se com essa pesquisa a predominância de pesquisas realizadas com ênfase no Ensino Superior e a baixa ocorrência de pesquisas, com essa ênfase, na Educação Básica. Não foram mapeadas pesquisas com ênfase no Ensino Médio e apenas uma das pesquisas aborda 0 uso da modelagem matemática atrelado às tecnologias digitais no Ensino Fundamental.

Para além dos objetivos abordados neste trabalho, outras pesquisas poderiam ser feitas, no que tange a Modelagem Matemática e o uso de TDE, de modo a analisar os trabalhos que desenvolveram suas pesquisas na modalidade Educação a Distância (EAD) em plataformas de ambientes virtuais. Além disso, um maior enfoque nas contribuições das TDE no desenvolvimento de atividades de Modelagem Matemática poderá ser feito em futuras pesquisas, com vistas a compreensão do seu uso na representação, interpretação e assimilação de conhecimentos matemáticos. 


\section{Referências}

ARAÚJO, J. de L. Cálculo, Tecnologias e Modelagem Matemática: as discussões dos alunos. Tese de doutorado. Universidade Estadual Paulista (UNESP). Programa de PósGraduação em Educação Matemática. Instituto de Geociências e Ciências Exatas, 2002.

ALMEIDA, L. M. W.; SILVA, K. P.; VERTUAN, R. E. Modelagem Matemática na Educação Básica. São Paulo: Contexto, 2012.

; PALHARINI, B. N. Os" Mundos da Matemática" em Atividades de Modelagem Matemática. Boletim de Educação Matemática, v. 26, n. 43, p. 907-934, 2012.

BARBOSA, J. C. Modelagem Matemática: O que é? Por que? Como? Veritati, n. 4, p. 73-80, 2004.

BASSANEZI, R. C. Ensino-aprendizagem com modelagem matemática. 1. ed., São Paulo: Editora Contexto, 2002.

BEAN, D. A modelagem de monteiro lobato: o caso da fábula a cigarra e a formiga. Em: Conferência Nacional sobre Modelagem na Educação Matemática, 9., 2015, São Carlos. Anais..., São Carlos: Sbem, p. 1 - 16, 2015.

BISOGNIN, E.; BISOGNIN, V.; Percepções de Professores sobre o Uso da Modelagem Matemática em Sala de Aula. Boletim de Educação Matemática, Rio Claro, v. 26, n. 43, p.1049-1079, ago. 2012.

BORBA, M. C.; CHIARI, A. S. S. Diferentes Usos de Tecnologias Digitais nas Licenciaturas em Matemática da UAB. Nuances: estudos sobre Educação, Presidente Prudente-SP, v. 25, n. 2, p. 127-147, maio/ago. 2014.

BORSSOI, A. H. Modelagem matemática, aprendizagem significativa e tecnologias: articulações em diferentes contextos educacionais. 2013. 256 f. Tese (Doutorado) - Curso de Doutorado em Ensino de Ciências e Educação Matemática), Centro de Ciências Exatas, Universidade Estadual de Londrina, Londrina, 2013.

BONOTTO, D. L.; SCHELLER, M.; BIEMBENGUT, M. S. Modelagem nas ciências e matemática: percepção de professores em formação continuada. Em: Conferência Nacional sobre Modelagem na Educação Matemática, IX, São Carlos: Anais, p. 1-15, 2015.

BRASIL, MEC, SEB. Orientações curriculares para o ensino médio. Ciências da natureza, matemática e suas tecnologias. Brasília: MEC, SEB, 2006.

BRANDT, C. F.; BURAK, D.; KLÜBER, T. E. Modelagem Matemática: uma perspectiva para a Educação Básica. Ponta Grossa: Editora UEPG, 148 p., 2010.

BURAK, D. Modelagem matemática: ações e interações no processo de ensinoaprendizagem. 1992. 130 f. Tese (Doutorado) - Programa de pós-graduação em educação, Faculdade de Educação, Universidade Estadual de Campinas, Campinas, 1992.

Modelagem Matemática e a sala de aula. Em: Encontro Paranaense de Modelagem em Educação Matemática, 1., 2004, Londrina. Anais... Londrina: UEL, 2004. p. 110, 2004.

DELLA VECCHIA, R. A Modelagem Matemática e a Realidade do Mundo Cibernético. 2012. 275 f. Tese (Doutorado) - Curso de Doutorado em Educação Matemática, 
Instituto de Geociências e Ciências Exatas, Universidade Estadual Paulista Júlio de Mesquita Filho, Rio Claro, 2012.

KENSKI, V. M. Educação e tecnologias: o novo ritmo da informação. Campinas: Papirus, 2007.

MALHEIROS, A. P. dos S. A produção matemática dos alunos em um ambiente de modelagem. 2004. 180 f. Dissertação (Mestrado) - Curso de Instituto de Geociências e Ciências Exatas Campus de Rio Claro, Universidade Estadual Paulista, Rio Claro, 2004.

OLIVEIRA, G. P.; MARCELINO, S. B. Estratégias didáticas com o software Superlogo: adquirir fluência e pensar com tecnologias em Educação Matemática. Educação Matemática Pesquisa, v. 17, n. 4, p. 816-842, 2015.

PARANHOS, M. de M. Parametrização e Movimentação de Curvas e Superfícies para uso em Modelação Matemática. 2015. 154 f. Tese (Doutorado) - Curso de Doutorado em Educação Matemática, Pontifícia Universidade Católica de São Paulo, São Paulo, 2015.

PARANÁ. Secretaria de Estado da Educação do Paraná. Superintendência da Educação. Diretrizes Curriculares da Educação Básica: Matemática. Curitiba: SEED, p. 1-81, 2008.

PEREIRA, R. dos S. G.; DOS SANTOS JÚNIOR, G. Modelagem Matemática e o Ensino de Ajuste de Funções: um caderno pedagógico. Boletim de Educação Matemática, v. 27, n. 46, p. 531, 2013.

SALANDINI, E. J. de A. A Modelagem Matemática na Introdução do Conceito de Equação para alunos de sétimo ano do Ensino Fundamental. 2011. 110 f. Dissertação (Mestrado) Curso de Mestrado Profissional em Ensino de Matemática, Pontifícia Universidade Católica de São Paulo, São Paulo, 2011.

SEKI, J. T. P.; SILVA, A. C.; PEREIRA, R. S. G. Formação continuada e modelagem matemática: um estudo dos anais de eventos da educação matemática. Em: Encontro Nacional de Educação Matemática, 13., 2016, São Paulo. Anais... São Paulo: UNICSUL, p. $1-$ 13, 2016.

SOARES, D. da S. Uma Abordagem Pedagógica Baseada na Análise de Modelos para Alunos de Biologia: qual o papel do software? 2012. $341 \mathrm{f}$. Tese (Doutorado) - Curso de Doutorado em Educação Matemática, Instituto de Geociências e Ciências Exatas, Universidade Estadual Paulista Júlio de Mesquita Filho, Rio Claro, 2012.

KITCHENHAM, B. Procedures for Performing Systematic Reviews. Keele UK Keele University- Publisher: Citesser, 33(TR/SE-0401), 28, 2004.

VERTUAN, R. E. Um olhar sobre a modelagem matemática à luz da teoria dos registros de representação semiótica. 2007. 140 f. Dissertação (Mestrado) - Curso de Mestrado em Ensino de Ciências e Educação Matemática, Universidade Estadual de Londrina, Londrina, 2007.

Submissão: $20 / 11 / 2016$

Aceite: 19/06/2017 